\title{
artigo
}

Dias, A.R.P.; Vitor, T.F.N.; Anunciação, T.W.Q.; Carvalho, D.J.M.; Canavarro, D.A.; Fernandes, L.J.;

Perfil da enfermeira no acolhimento com classificação de risco em uma emergência obstétrica

\section{Perfil da enfermeira no acolhimento com classificação de risco em uma emergência obstétrica}

\author{
Nurse Profile in the classification of risk in obstetric emergency \\ Perfil de la enfermera de acogimiento con clasificación de riesgo en una emergencia obstétrica
}

\begin{abstract}
RESUMO
Objetivo: identificar o perfil das enfermeiras atuantes em uma maternidade pública no acolhimento com classificação de risco de uma emergência obstétrica. Método: trata-se de uma pesquisa descritiva e exploratória, realizada entre os meses de outubro e novembro de 2020 por meio de um questionário online. Resultados: ao analisar o questionário respondido pelas enfermeiras que participaram do estudo atuantes no setor de Acolhimento com Classificação de Risco da referida maternidade, é perceptível que o público é predominantemente de mulheres jovens, pretas e pardas, com média de 38 anos de idade e oriundas de faculdades do ensino privado da cidade de Salvador Bahia, com contrato de trabalho terceirizado, e 62,5\% com duplo vínculo. Conclusão: este perfil possibilita identificar o desenho dos profissionais atuantes nas salas de classificação de risco nas emergências obstétricas e pode ser o caminho de apontar a realidade da precarização dos vínculos nos serviços públicos de saúde. DESCRITORES: Descrição de Cargo; Emergências; Enfermagem; Enfermagem Obstétrica; Papel do Profissional de Enfermagem.
\end{abstract}

\section{ABSTRACT}

Objective: to identify the profile of nurses working in a public maternity hospital at the reception with risk classification of an obstetric emergency. Method: this is a descriptive and exploratory research, carried out between the months of October and November 2020 through an online questionnaire. Results: when analyzing the questionnaire answered by the nurses who participated in the study working in the Reception sector with Risk Classification of maternity, it is noticeable that the public is predominantly of young, black and brown women, with an average of 38 years of age and from private education colleges in the city of Salvador Bahia, with an outsourced labor contract, and $62.5 \%$ with a double bond. Conclusion: this profile makes it possible to identify the design of professionals working in the risk classification rooms in obstetric emergencies and may be the way to point out the reality of the precariousness of bonds in public health services.

DESCRIPTORS: Job Description; Emergencies; Nursing; Obstetric Nursing; Nurse's Role.

\section{RESUMEN}

Objetivo: identificar el perfil de enfermeras que laboran en una maternidad pública en la recepción con clasificación de riesgo de emergencia obstétrica. Método: se trata de una investigación descriptiva y exploratoria, realizada entre los meses de octubre y noviembre de 2020 a través de un cuestionario online. Resultados: al analizar el cuestionario respondido por las enfermeras que participaron del estudio laborando en el sector de Recepción con Clasificación de Riesgo de la maternidad, se nota que el público es predominantemente de mujeres jóvenes, negras y morenas, con un promedio de edad de 38 años. y de los colegios privados de educación de la ciudad de Salvador Bahía, con contrato laboral tercerizado, y el 62,5\% con doble vínculo. Conclusión: este perfil permite identificar el diseño de los profesionales que laboran en las salas de clasificación de riesgo en emergencias obstétricas y puede ser la forma de señalar la realidad de la precariedad de los vínculos en los servicios públicos de salud.

DESCRIPTORES: Perfil Laboral; Urgencias Médicas; Enfermería; Enfermería Obstétrica; Rol de la Enfermera.

RECEBIDO EM: 04/06/2021 APROVADO EM: 11/06/2021

\section{Aimée Rosa Paranhos Dias}

Graduanda do Curso de Enfermagem do Centro Universitário Jorge Amado- UNIJORGE. Ba.

ORCID: 0000-0001-6051-5469

\section{Thaís Fernanda Nascimento Vítor}

Graduanda do Curso de Enfermagem do Centro Universitário Jorge Amado-UNIJORGE. Ba.

ORCID: 0000-0002-8728-5891 
Thales William Queiroz da Anunciação

Graduando do Curso de Enfermagem do Centro Universitário Jorge Amado-UNIJORGE. Ba.

ORCID: 0000-0002-8841-4659

\section{Danuza Jesus Mello de Carvalho}

Professora assistente da coordenação do curso de enfermagem, Centro Universitário Jorge Amado, Mestre em Enfermagem e Saúde; Enfermeira Intensivista e Nefrologista.

ORCID: 0000-0002-0851-0836

\section{Danielle de Andrade Canavarro}

Coordenadora Curso de Enfermagem; Centro Universitário Jorge Amado, Mestra em Enfermagem; Enfermeira Obstétrica e Emergencista.

ORCID: 0000-0002-5346-6651

\section{Lucas Jesus Fernandes}

Mestrando do Programa de Pós Graduação em Enfermagem e Saúde; Universidade Federal da Bahia, Enfermeiro.

ORCID: 0000-0002-7657-8314

\section{INTRODUÇÃO}

A grande demanda por atendimento aos serviços de saúde nas unidades de urgência e emergência no Brasil, revelam a importância que gerou a reflexão sobre a relevância do desenvolvimento e qualidade das triagens no atendimento à população. $\mathrm{O}$ acolhimento com classificação de risco (ACCR) foi desenvolvido como estratégia para aperfeiçoar $o$ atendimento nas unidades de urgência e emergência, otimizar a assistência, minimizar possíveis complicações causadas aos pacientes por superlotação dos serviços de saúde sendo a porta de entrada para o serviço estabelecendo uma relação de confiança entre profissional e cliente ${ }^{1}$.

O ACCR possibilita o direcionamento do paciente para a área de tratamento e/ou especialidade médica mais adequada à sua necessidade, sendo as enfermeiras profissionais habilitadas para a atuação na sala de classificação, através da consulta de enfermagem, para este fim, a enfermeira com base em um protocolo preconizado pela instituição classifica $o$ usuário utilizando um sistema de cores ${ }^{2}$.

Tratando-se de uma emergência obstétrica, o ACCR apresenta especificidades únicas às necessidades e às demandas relativas ao processo gravídico, que muitas vezes podem mascarar situações de emergências clínicas que demandam habilidades de profissionais devidamente capacitados.
A grande demanda por atendimento

aos serviços de

saúde nas unidades

de urgência e

emergência no

Brasil, revelam a

importância que

gerou a reflexão

sobre a relevância

do desenvolvimento

e qualidade

das triagens no

atendimento à

população.
Um dos importantes fatores a serem observados é a qualificação profissional, pois a política de Acolhimento com Classificação de Risco compreende a importância da formação e experiência no atendimento em urgência/emergência, o que garante uma assistência mais eficaz, humana e com sensibilidade clínica apu$\operatorname{rada}^{3}$. Desta forma, a qualificação profissional impacta na qualidade do serviço, no tempo de espera e repercute em melhores resultados no atendimento das enfermeiras e na recuperação da saúde das gestantes que aguardam o atendimento.

Diante da relevância dos serviços prestados pelas enfermeiras nas salas de ACCR das emergências obstétricas, fez emergir os seguintes questionamentos: Quem são as enfermeiras atuantes na sala de ACCR da emergência obstétrica de uma maternidade pública de Salvador? Qual tipo de formação essa profissional possui? Qual tipo de vínculo profissional essas enfermeiras possuem na instituição a que pertencem? Este estudo teve como objetivo identificar o perfil das enfermeiras atuantes no acolhimento com classificação de risco de uma emergência obstétrica de uma maternidade pública de Salvador.

\section{MÉTODO}

Pesquisa exploratória de abordagem quantitativa realizada no Estado da Bahia, 
Tabela 1. Distribuição das Enfermeiras por perfil sócio demográfico, Salvador, BA, Brasil, 2021.

\begin{tabular}{|c|c|c|}
\hline \multirow{2}{*}{ FAIXA ETÁRIA } & $\mathrm{N}$ & $\%$ \\
\hline & 16 & 100,0 \\
\hline $26-34$ & 6 & 37,5 \\
\hline $35-40$ & 4 & 25,0 \\
\hline $41-46$ & 3 & 18,7 \\
\hline $47-57$ & 3 & 18,7 \\
\hline SEXO & 16 & 100,0 \\
\hline Feminino & 14 & 87,5 \\
\hline Masculino & 2 & 12,5 \\
\hline RAÇA & 16 & 100,0 \\
\hline Preto & 6 & 37,4 \\
\hline Pardo & 5 & 31,3 \\
\hline Branco & 5 & 31,3 \\
\hline Indígena & 0 & 0 \\
\hline Amarela & 0 & 0 \\
\hline Fonte: dados da pesquisa. & & \\
\hline
\end{tabular}

Tabela 2. Distribuição das Enfermeiras por Qualificação Profissional, Salvador, BA, Brasil, 2021.

\begin{tabular}{|c|c|c|}
\hline \multirow{2}{*}{ PÓS GRADUAÇÃO } & $\mathrm{N}$ & $\%$ \\
\hline & 16 & 100,0 \\
\hline $\operatorname{Sim}$ & 16 & 100,0 \\
\hline Não & 0 & 0 \\
\hline Especialização & 14 & 87,4 \\
\hline Mestrado & 1 & 6,3 \\
\hline Doutorado & 0 & 0 \\
\hline Nenhum & 1 & 6,3 \\
\hline \multicolumn{3}{|l|}{ ÁREA DE ESPECIALIZAÇÃO } \\
\hline Obstetrícia & 3 & 18,8 \\
\hline Emergência & 2 & 12,5 \\
\hline UTI & 4 & 25,0 \\
\hline Outros & 7 & 43,7 \\
\hline \multicolumn{3}{|l|}{ Fonte: dados da pesquisa. } \\
\hline \multicolumn{3}{|c|}{$\begin{array}{l}\text { Tabela 3. Distribuição das Enfermeiras participantes por Perfil Profissional } \\
\text { Salvador, BA, Brasil, } 2021 .\end{array}$} \\
\hline \multirow{2}{*}{ TIPO DE VÍNCULO } & $\mathrm{N}$ & $\%$ \\
\hline & 16 & 100,0 \\
\hline Estatutário & 1 & 6,2 \\
\hline CLT & 15 & 93,8 \\
\hline
\end{tabular}

município de Salvador com as enfermeiras atuantes na sala de ACCR de uma maternidade pública de Salvador. Os critérios de inclusão adotados foram: as participantes atuassem na sala com classificação de risco e com mais de seis meses de atuação na referida maternidade. Foram excluídos da pesquisa os sujeitos que: enfermeiras que estavam afastadas por licença médica ou de férias, que não completaram o preenchimento total e correto do instrumento ou estavam impossibilitadas em responder o questionário, e por fim aquelas que não foi possível localizar o contato telefônico com registro em aplicativo de mensagem instantânea.

A coleta foi iniciada no segundo semestre de 2020 entre os meses de outubro e novembro, por meio da aplicação de um questionário disponibilizado no aplicativo Google Docs ', sendo encaminhado através do e-mail e telefone de contato previamente solicitado. Este possuía duas partes: uma que dizia respeito ao perfil sociodemográfico da participante com dez questões, e outra sobre os dados de formação e perfil profissional com nove questões.

A interpretação dos dados coletados foi feita pela estatística descritiva no qual foram organizados e analisados por intermédio de uma planilha eletrônica em seguida discutida entre os autores. Quanto aos procedimentos éticos, a presente pesquisa foi aprovada pelo Comitê de Ética em Pesquisa da Universidade Católica do Salvador (CEP/UCSal) sob o parecer n. 3.791 .490 e CAEE 25933119.0.0000.5628. As participantes assinaram o Termo de Consentimento Livre e Esclarecido (TCLE), respeitando a Resolução n. ${ }^{\circ}$ 466/12 do Conselho Nacional de Saúde (CNS), que disserta sobre a pesquisa com seres humano dando o consentimento de forma virtual após a leitura, no qual só era possível dar continuidade ao processo após o aceite.

\section{RESULTADOS}

Das 16 enfermeiras atuantes na sala de ACCR na maternidade estudada, foi enviado por mensagem através do apli- 


\section{1}

2

Mais

FAIXA SALARIAL

1 a 2 salários-mínimos

3 a 4 salários-mínimos

5 a 6 salários-mínimos

Acima de 6 salários-mínimos

Fonte: dados da pesquisa

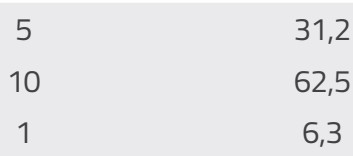

$\begin{array}{ll}7 & 43,7\end{array}$

425,0

425,0

16,3

cativo de mensagem instantânea o instrumento de coleta digital (ICD) para as enfermeiras que atenderão o critério de inclusão $(n=16)$. Assim foram analisadas 16 enfermeiras da maternidade em questão. A tabela 1 descreve a caracterização das enfermeiras por perfil sócio demográfico.

No que tange a formação, a tabela a seguir elucida os percentuais referente as variáveis estudadas:

Referente ao perfil profissional a tabela 3 apresenta os achados encontrados:

\section{DISCUSSÃO}

Quanto as participantes do estudo, houve predominância no sexo feminino e a faixa etária apresentou variação entre 26 e 56 anos de idade. Esses achados corroboram diversos estudos na área, mesmo com a nova tendência de alguns estudos apresentarem o rejuvenescimento e um aclive na masculinizarão da profissão $^{4-6}$.

A predominância na enfermagem do sexo feminino encontrado no estudo é esperada e compartilhada por outros pesquisadores. Estudo sobre perfil de enfermeiras em Cabo Verde - África, elucida que tal perfil é justificado pela feminilização cultural da profissão atribuído o cuidado a uma ação inerente ao gênero feminino, relação ainda persistente atualmente ao processo do cuidado na sociedade, mantendo conexões da participação feminina na Enfermagem ${ }^{7}$.

Quanto à raça/cor, nota-se a maioria autodeclarada como preta e parda $(68,7 \%)$. O acesso à qualificação profis-

\section{Quanto as}

participantes do

estudo, houve

predominância

no sexo feminino

e a faixa etária

apresentou variação

entre 26 e 56 anos

de idade. Esses

achados corroboram

diversos estudos na

área, mesmo com

a nova tendência

de alguns estudos

apresentarem o

rejuvenescimento

e um aclive na

masculinizarão da

profissão. sional está intimamente relacionado às possibilidades que indivíduos de diferentes grupos étnicos e níveis de renda têm na sociedade. Desde a época em que a enfermagem brasileira foi conceituada como moderna até hoje, observa-se empenho de segmentos da sociedade civil organizada em desenvolver políticas públicas que reparassem o longo período em que o acesso da população negra à educação foi restrito ${ }^{8}$.

No que concerne à formação acadêmica, a maioria são oriundas de instituiçôes de ensino superior privado. Esse dado reflete as ações e incentivo das políticas públicas do governo brasileiro nos anos 2000, que teve como objetivo viabilizar o acesso de diversas camadas sociais nas universidades públicas e privadas, através tanto com a implantação de cotas e bolsas de estudo parciais ou integrais quanto na ampliação do setor privado e/ou possibilidade do financiamento estudantil ${ }^{9}$.

Quanto à formação nota-se expressivo percentual (94,2\%) de enfermeiras já cursaram ou cursam algum tipo de pós-graduação. Destaca-se o grande contingente de profissionais que fizeram especialização, destes, 31,3\% possui formação especializada na área de emergência e obstetrícia. Esta informação é um importante achado, pois a política de Acolhimento com Classificação de Risco preconiza que os enfermeiros preferencialmente tenham qualificação e/ou experiência em serviço de urgência e emergência. A enfermeira do ACCR deve ter ação rápida, isso exige expertise, destreza e habilidade com capacidade de julgamento clínico preciso $^{2}$.

Corroborando com o achado desta pesquisa, outro estudo realizado em Cabo Verde, a política regulamentadora da profissão vem exigindo uma melhor formação para os profissionais, incentivando, assim, a busca pela qualificação técnica e científica dos enfermeiros com objetivo da melhoria na qualidade dos serviços ${ }^{7}$.

Em termos de vínculo empregatício, no setor específico estudado, 93,8\% das enfermeiras apresentaram relação de tra- 


\section{artigo}

Dias, A.R.P.; Vitor, T.F.N.; Anunciação, T.W.Q.; Carvalho, D.J.M.; Canavarro, D.A.; Fernandes, L.J.;

Perfil da enfermeira no acolhimento com classificação de risco em uma emergência obstétrica

balho terceirizado. Essa nova conjuntura dos hospitais públicos é uma estratégia de reposição de pessoal de uma suposta e não comprovada ideia de melhoria da prestação de serviços, que apresenta diversas consequências para a gestão dos serviços de saúde como: instabilidade empregatícia, alta rotatividade de pessoal, descontinuidade dos processos de trabalho, ataque aos direitos trabalhistas, ao padrão salarial e às condições de trabalho ${ }^{10}$.

Ao analisar vencimento salariais das enfermeiras atuantes no setor estudado, foi identificado uma variação ampla nesta variável, com valor médio de $\mathrm{R} \$ 3.300,00$. Este dado faz refletir sobre a ausência de legislação que assegure às trabalhadoras do campo da enfermagem um piso salarial e jornada de trabalho, o que, associado à frágil organização política, as expõe aos interesses dos empregadores e as deixam suscetíveis a diferentes vencimentos sejam ele no setor público ou privado.

Estudo realizado em 2015 sobre os vencimentos pagos às enfermeiras, foi identificado que a remuneração média para enfermeiros foi entre $\mathrm{R} \$ 3.999,17 \mathrm{e}$ $\mathrm{R} \$ 4.030,67^{11}$. Esse dado em comparação com o presente estudo identifica que a média geral salarial de enfermeiras com vínculo empregatício terceirizado, não está equiparada. O elevado número de salários entre um até dois salários-mínimos reverbera uma predominância de baixos salários pagos às enfermeiras, conforme aponta estudo do International Council of Nurses ${ }^{12}$. Os baixos vencimentos das enfermeiras na rede de serviços públicos associados à terceirização podem significar uma forma clara da precarização do trabalho da enfermeira.

\section{CONCLUSÃO}

Identificar o perfil das enfermeiras atuantes na ACCR e reconhecê-las por meio desse estudo, viabiliza a possibilidade de novos horizontes que contribuam para repensar na gestão e no perfil profissional dos serviços de saúde e desvelam um conjunto de elementos que podem ser aprofundados a partir de outras pesquisas, a fim de explorar categorias de análise tanto do processo de trabalho quanto da precarização que não foram contemplados neste estudo.

\section{REFERÊNCIAS}

1. Oliveira JLC, Gatti AP, Barreto MS, Bellucci Junior JA, Góes HLF, Matsuda LM. Acolhimento com classificação de risco: percepções de usuários de uma Unidade de Pronto Atendimento. Texto \& contexto enferm. [Internet]. 2017 [acesso em 2021 jan 26];26(1):e0960014. Disponivel em: https://doi. org/10.1590/0104-07072017000960014

2. Duro CLM, Lima MADS, Weber LAF. Opinião de enfermeiros sobre classificação de risco em serviços de urgência. REME rev. min. enferm. [Internet]. 2017 [acesso 2021 jan 25];21:e-1062. Disponivel em: https://doi.org/10.5935/1415-2762.20170072

3. Figueiroa MN, Menezes MLN, Monteiro EMLM, Aquino JM, Mendes NOG, Silva PVT. Acolhimento do usuário e classificação de risco em emergência obstétrica: avaliação da operacionalização em maternidade-escola. Esc Anna Nery [Internet]. 2017 [acesso 2021 jan 25];21(4):e20170087. Disponivel em: https:// doi.org/10.1590/2177-9465-EAN-2017-0087

4. Nogueira LDP, Mamede FV, Souza L. Perfil dos profissionais de enfermagem que atuam na assistência pré-natal: um estudo descritivo. Rev. enferm. UFPE on line. [Internet]. 2016 [acesso 2021 jan 26];10(Supl. 2):762-70. Disponivel em: https://doi. org/10.5205/reuol.6884-59404-2-SM-1.1002sup201610

5. Machado MH, Aguiar Filho W, Lacerda WF, Oliveira E, Lemos $\mathrm{W}$, Wermelinger $\mathrm{M}$, et al. Características gerais da enfermagem: o perfil sócio demográfico. Enferm. Foco [Internet]. 2015 [acesso 2021 jan 26];7(ESP):09-14. Disponível em: https://doi. org/10.21675/2357-707X.2016.v7.nESP.686

6. Marziale MHP, Hong OS, Morris JA, Rocha FLR. Atribuições e funções dos enfermeiros do trabalho no Brasil e nos Estados Unidos. Rev. Latino-Am. Enfermagem [Internet]. 2010 [acesso 2021 jan 18];18(2):182-88. Disponivel em: http://dx.doi. org/10.1590/S0104-11692010000200007
7. Semedo DSR, Pelzer MT, Ventura J, Paula SF, Moniz ASB, Semedo JAC. Profile of nurses from Cape Verde/Africa: the influencing factors of life quality. Rev. Pesqui. (Univ. Fed. Estado Rio J., Online) [Internet]. 2018 [acesso 2021 jan 18];10(4):945-950. Disponivel em: http://dx.doi.org/10.9789/2175-5361.2018. v10i4.945-950

8. Oliveira MLP. A presença negra na Enfermagem [Internet]. 2019 [acesso 2021 jan 20]. Disponivel em: http://sergs.org.br/ artigos/a-presenca-negra-na-enfermagem/\#: :text=Desde\%20 a $\% 20 \%$ C3\%A9poca $\% 20$ em $\% 20$ que,negra $\% 20 \%$ C3\%A0\%20educa\%C3\%A7\%C3\%A30\%2C\%20foi\%20negado

9. Aguiar V. Um Balanço das Políticas do Governo Lula para a Educação Superior: continuidade e ruptura. Rev. Sociol. Polit. [Internet]. 2016 [acesso 2021 jan 18];24(57):113-126. Disponível em: https://doi.org/10.1590/1678-987316245708

10. Marques FC. Gestão dos Serviços de Enfermagem frente a terceirização em hospitais públicos: mediando conflitos [dissertação]. Rio de Janeiro: Universidade Estadual do Rio de Janeiro, Faculdade de Enfermagem; 2019 [acesso em 2021 jan 18]. Disponivel em: http://www.bdtd.uerj.br/tde_busca/arquivo. php?codArquivo $=17038$

11. Lombardi MR, Campos VP. A Enfermagem no Brasil e os contornos de gênero, raça/cor e classe social na formação do campo profissional. Revista da ABET [Internet]. 2018 [acesso 2021 jan 20];17(1):28-46. Disponivel em: https://doi.org/10.22478/ufpb.1676-4439.2018v17n1.41162

12. Spetz J. Unemployed and Underemployed Nurses [Internet]. International Centre for Human Resources in Nursing. Geneva: International Council of Nurses; 2011 [acesso 2021 jan 26]. 51p. Disponivel em: https://www.hrhresourcecenter.org/node/4006. html 